\title{
CASE \\ REPORT
}

GMJ.2016;5(2):98-102

Received: 2016-05-3

Revised: 2016-05-13

Accepted: 2016-05-25

\section{Dual Ectopic Multinodular Goiter: A Case Report}

\author{
Masoumeh Saeedi ${ }^{1}$, Mohammad Hossein Khosravi ${ }^{2,3}$ 凹 \\ ${ }^{1}$ Department of Otorhinolaryngology-Head and Neck Surgery, Faculty of Medicine, Baqiyatallah University of Medical Sciences, \\ Tehran, Iran \\ ${ }^{2}$ Students' Research Committee (SRC), Baqiyatallah University of Medical Sciences, Tehran, Iran \\ ${ }^{3}$ International Otorhinolaryngology Research Association (IORA), Universal Scientific Education and Research Network (USERN), \\ Tehran, Iran
}

\begin{abstract}
Background: As the first endocrine gland to form during embryogenesis, Thyroid develops in the 7th embryonic week through migration from foramen cecum to its adult position, anterolateral of 2nd and 4th tracheal cartilages. Ectopic Thyroid can be found anywhere along the migration path with or without co-existence of a normally located Thyroid. Ectopic thyroid glands at two different locations are so rare that only about 24 cases of dual ectopia have been reported. We present the first case of dual ectopic submandibular multinudolar goiter.Case Report: We describe a 35-year-old housewife who consulted us complaining two huge and gradually-growing swellings in right and left upper neck regions. In physical examination, two great well-defined masses, one in right and one in left submandibular regions, were seen which had firm formidability with no mobility, tenderness or evidence of inflammation. Imaging and fine-needle aspiration confirmed the diagnosis of dual ectopic submandibular multinodular goiter in our patient as the first case of occurrence.Conclusion: Finally, we recommend ectopic thyroid to be considered in the diagnosis of laterally located and submandibular neck masses especially in the absence of normally located thyroid. Additionally, we confirmed the idea that not all the laterally-located ectopic thyroids are malignant.[GMJ.2016;5(2):98-102]
\end{abstract}

Keywords: Ectopic Thyroid; Nodular Goiter; Multinodular Goiter

\section{Introduction}

A $\mathrm{s}$ the first endocrine gland to form during embryogenesis, Thyroid develops in the 7th embryonic week through migration from foramen cecum to its adult position, anterolateral of 2nd and 4th tracheal cartilages [1]. Ectopic Thyroid can be found anywhere along the migration path with or without co-existence of a normally located Thyroid. In 90 percent of cases, it is located

\section{GMJ}

2016 Galen Medical Journal

Fax: +98 7312227091

PO Box 7461686688

Email:info@gmj.ir in the base of the tongue and in the remaining 10 percent, it is identified with other anatomic locations such as larynx, pharynx, esophagus, mediastinum and submandibular. Multinodular goiter (MNG) is defined as the enlargement of the thyroid gland with formation of nodules which has a total prevalence of 4.7$37.3 \%$ worldwide [2]. Ectopic thyroid can be involved by the same pathologies as the normally located one such as thyrotoxicosis or malignancy as well as MNG.

\footnotetext{
Correspondence to:

Mohammad Hossein Khosravi, Students' Research

Committee (SRC), Baqiyatallah University of Medical

Sciences, Tehran, Iran

Telephone number: +989126441288

Email Address: dr.mhkhosravi@gmail.com
} 
Ectopic thyroid glands at two different locations are so rare that only about 24 cases of dual ectopia have been reported [3]. We present the first case of dual ectopic submandibular MNG. Informed consent was obtained from patient included in the study.

\section{Case Presentation}

We describe a 35-year-old housewife who consulted us complaining two huge and gradually-growing swellings in right and left upper neck regions. During pregnancy and lactation period, the swellings had a significant progress.

She was clinically euthyroid except with a history of constipation during pregnancy. In physical examination, two great well-defined masses; one in right and the other in left submandibular regions, were seen which had firm formidability with no mobility, tenderness or evidence of inflammation (Figure-1). Thyroid function test (TFT) was among normal ranges $(\mathrm{TSH}=2.27 \mathrm{mIU} / \mathrm{L})$. Past medical history of the patient revealed no evident disease or hospitalization except one cesarean section

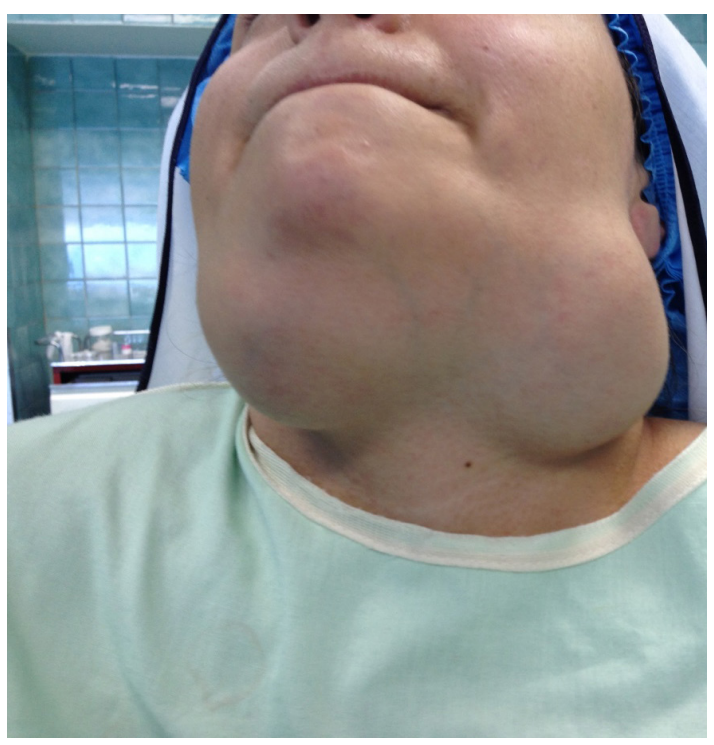

Figure 1. Right and left submandibular swellings in the patient surgery. Gastrointestinal cancer in her father was the only positive family history.

After physical examination, the patient was admitted for further evaluations. Axial neck computed topography (CT) scan, magnetic resonance imaging (MRI) (with and without contrast) and sonography were requested for her as well as fine needle aspiration (FNA). CT scan showed a huge lobulated mass with defined margin sized $130 \times 60$ millimeters with severe heterogeneous enhancement (Figure-2). Normal thyroid tissue was not seen in $\mathrm{CT}$ scan and the mentioned mass was suggested to be from thyroid origin based on CT scan report. Ultrasound evaluation revealed a double-lobe heterogeneous mass sized $130 \times 60$ millimeters with pressure effect on regional elements. Normal thyroid tissue was not seen in its anatomic place by ultrasound either. MRI revealed a very large and well circumscribed bilateral mass lesion sized $135 \times 55$ millimeters with heterogeneous signal on all sequences and mass effect on anterior side of pharynx at superior anterior part of neck in-

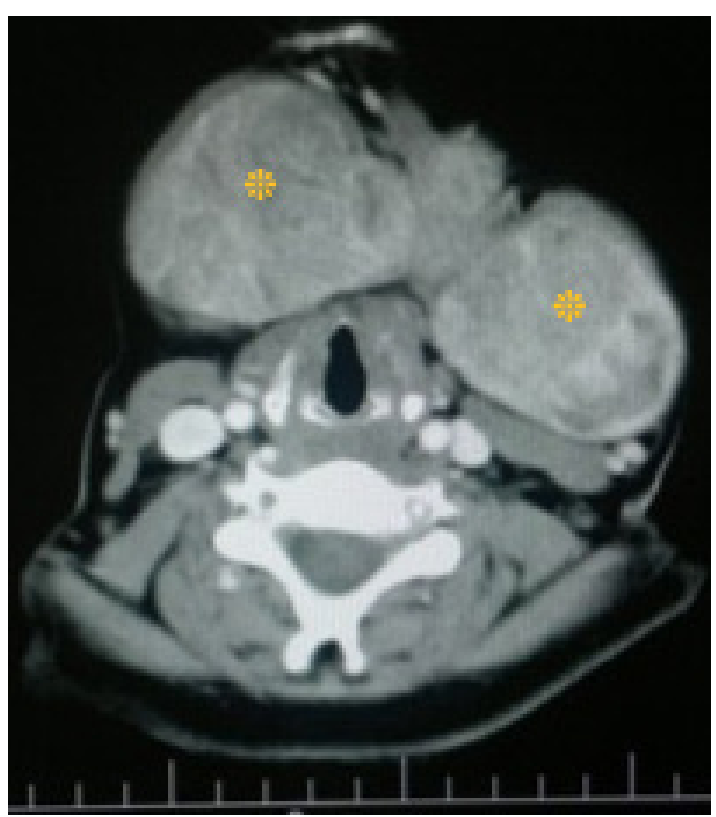

Figure 2. Axial image of contrast-enhanced CT scan showing the localization of left and right submandibular goiter (stars) 


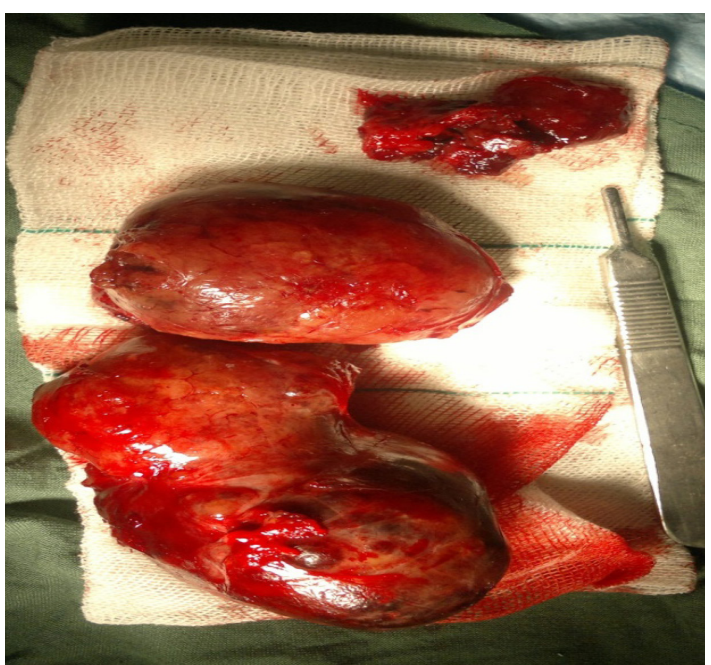

Figure 3. Postoperative images from extracted masses

dicating intense enhancement on post Gadolinium images. Normal thyroid gland tissue was not seen in MRI. FNA was suggestive of hyperplastic multinodular goiter. Radioactive iodine uptake test (RAIU) showed an uptake similar to two huge thyroid lobes.

Based on the aforementioned tests and imaging, the patient was candidate for surgery with diagnosis of MNG. Patient underwent midline neck incision from left sternocleidomastoid (SCM) muscle to right SCM. SCM muscle was elevated from the mass which was stuck to the hyoid bone by an isthmus. Therefore, the body of hyoid bone was removed with whole mass, which spread to the floor of the mouth, by Sistrunk method. Extracted masses (Figure-3) were sent for pathology assessment which confirmed the ectopic multinodular goiter.

Three hours after surgery, the patient had nausea associated with one attack of vomiting. The vital signs were stable. The day after surgery, Calcium and Phosphorus levels were 9.5 and $3.1 \mathrm{mg} / \mathrm{dl}$, respectively. Also, procalcitonin (PCT) activity was reported $0.24 \%$. The patient was discharged 3 days after surgery with appropriate general condition. Patient was referred to Endocrinologist for Thyroid hormone therapy. The three-year postoperative follow up was eventless.

\section{Discussion}

As the most common Thyroid development disorder, ectopic Thyroid has an incidence of $48-61 \%$, the real incidence has remained unknown though [4]. They are mostly asymptomatic since cadaver studies have suggested that $7-10 \%$ of adults may have thyroid tissue along the embryonic migration pathway [5]. Moreover, thyroid tissue has been found in parotid gland, trachea, mediastinum, heart, lung, duodenum, adrenal gland and even a more far location, uterus [6]. Submandibular region is another extremely rare reported location of aberrant thyroid tissue $[1,7]$. Ectopic thyroid glands at two different locations are so rare that only about 24 cases of dual ectopia have been reported [3]. Our case presented with a dual ectopic submandibular ectopic thyroid tissue.

Ectopic thyroids are able to play the role of a normal one; so they are potentially at the risk of similar thyroid pathologies such as inflammation, thyrotoxicosis, malignancy, calcification and MNG as in our case who was diagnosed to have dual ectopic MNG. Multinodular goiter is a slowly-progressive benign disease which has different clinical manifestations during its course, a dominant nodule at earlier and mass effect of enlarged goiter at later stage [2].

Ectopic thyroid tissue in lateral neck was first reported in the 18 th century and further reported cases were diagnosed to be malignant. Thus, previous data recognized laterally-located neck masses as "lateral aberrant thyroid 
tumors" because they were considered to be either a metastasis from thyroid carcinoma or primary tumors of aberrant thyroid [8]. Albeit, a considerable number of laterally situated benign thyroid ectopia have been reported so far such as our case who was diagnosed with MNG. Ectopic thyroid may present as laterally-located mass with or without a normal eutopic thyroid as in our case [4]. Kousta et al. have suggested that Ectopic thyroid tissue should be considered in the diagnosis of a cervical mass even in the presence of a eutopic thyroid gland [6]. Thyroid ectopia may be associated with hypo-function or hyper-function of thyroid. However, thyroid function tests were within normal limits in our patient as in Akamnu et al. case [4].

Thyroid ectopia will clinically manifest and be diagnosed in periods of physiologic stress and demand of thyroid hormones as in menopause and adolescence [9]; as the masses had significantly grown during pregnancy in our patient.

Agrawal et al. reported a 13-year old male complaining of swelling in the right upper neck for 7 months and normal routine laboratory data. Physical examination revealed a $4 \times 8 \mathrm{~cm}$ well-defined and non-tender submandibular mass. The patient underwent surgical excision of the mass and pathologic evaluation showed MNG[10]. Akanmu et al. reported a 34-year-old female with three left-sided neck masses which was diagnosed to be an enlarged multinodular left lobe of thyroid [4]. Although submandibular ectopic thyroid is rarely seen, it should be considered as a differential diagnosis of neck lumps. Furthermore, risk of metastasis or primary malignancy must be taken into account in such patients especially when there is a eutopic functional thyroid [7], as previous reports presented neck lumps were thought to be ectopic thyroid tissue but later found to be thyroid carcinoma metastases $[1,7]$.

To the best of our knowledge, the presented case is the first case of dual ectopic MNG. Regarding ectopic thyroid, location, size and the presence of symptoms or complications are determinative factors in treatment. The indications of treatment for MNG are suspected or confirmed malignancy, goiters with pressure effect, thyrotoxicosis, irritative symptoms or cosmetic concern [2]. Aside from mass effect reported by ultrasound, the chief complaint of our case was cosmetic concerns. Nearly all of the previous studies have suggested surgical excision of the mass in such patients as the treatment of choice $[6,10]$.

\section{Conclusion}

In conclusion, we described the first case of occurrence of dual ectopic submandibular multinodular goiter. We recommend ectopic thyroid to be considered in the diagnosis of laterally located and submandibular neck masses especially in the absence of normally located thyroid. Additionally, we confirmed the idea that not all laterally-located ectopic thyroids are malignant.

\section{Conflict of Interest}

Authors declare that they have no conflict of interest. 


\section{References}

1. Huang TS, Chen HY. Dual thyroid ectopia with a normally located pretracheal thyroid gland: case report and literature review. Head \& neck. 2007;29(9):885-8.

2. Lam S, Lang BH-H. A Review of the Pathogenesis and Management of Multinodular Goiter. Thyroid Disorders: Focus on Hyperthyroidism. 2014.

3. Chawla M, Kumar R, Malhotra A. Dual ectopic thyroid: case series and review of the literature. Clinical nuclear medicine. 2007;32(1):1-5.

4. Akanmu IN, Adewale OM. Lateral cervical ectopic thyroid masses with eutopic multinodular goiter: an unusual presentation. Hormones. 2009;8(2):150-3.

5. JJ S. Ectopic lingual thyroid. J Pathol. 1970;102:239-45.

6. Kousta E, Konstantinidis K, Michalakis C, Theodoropoulos GE, Vorias M, Georgiou M, et al. Ectopic thyroid tissue in the lower neck with a coexisting normally located multinodular goiter. Brief literature review. HORMONES-ATHENS-. 2005;4(4):231-4.
7. Choi J-Y, Kim J-H. Case of an ectopic thyroid gland at the lateral neck masquerading as a metastatic papillary thyroid carcinoma. Journal of Korean medical science. 2008;23(3):548-50.

8. Sweeney E, McDonnell L, O'BRIEN C. Medullary carcinoma of the thyroid presenting as tumours of the pharynx and larynx. Histopathology. 1981;5(3):263-75.

9. Toso A, Colombani F, Averono G, Aluffi P, Pia F. Lingual thyroid causing dysphagia and dyspnoea. Case reports and review of the literature. ACTA otorhinolaryngologica italica. 2009;29(4):213-7.

10. Agrawal R, Agrawal S, Gupta D, Gupta A. Ectopic multinodular Goitre-An unusual case. Indian Journal of Otolaryngology and Head and Neck Surgery. 2005;57(4):350-2. 